\title{
Iterative methods for solving absolute value equations
}

\author{
Rashid Ali ${ }^{\mathrm{a}, \mathrm{b}}$, Asad Alib,*, Shahid Iqbal ${ }^{\mathrm{b}}$ \\ a School of Mathematics and Statistics, HNP-LAMA, Central South University, Changsha 410083, Hunan, P.R. China. \\ ${ }^{b}$ Department of Mathematics, Abdul Wali Khan University, Mardan 23200, KPK, Pakistan.
}

\begin{abstract}
We suggest and analyze some iterative methods called Jacobi, Gauss-Seidel, SOR (successive over-relaxation), and modified Picard methods for solving absolute value equations $A x-|x|=b$, where $A$ is an $M$-matrix, $b \in R^{n}$ is a real vector, and $x \in R^{n}$ is unknown. Furthermore, we discuss the convergence of the suggested methods under suitable assumptions and represent their performance through our numerical results. Results are very encouraging and may stimulate further research in this direction.
\end{abstract}

Keywords: Absolute value equations, iterative methods, M-matrix, convergence, numerical experiments.

2020 MSC: 90C30, 65F10, 90C05.

(C2022 All rights reserved.

\section{Introduction}

Consider the absolute value equation (AVE) with matrix $A \in \mathrm{R}^{\mathrm{n} \times \mathrm{n}}$ is an M-matrix, $\mathrm{b} \in \mathrm{R}^{\mathrm{n}}$ and $|*|$ indicates the absolute value given by

$$
A x-|x|=b .
$$

In addition, the Eq. (1.1) is simplified form of the general AVE

$$
A x+B|x|=b,
$$

where $B \in R^{n \times n}$ was introduced by Rohn [32] and investigated in [23]. The AVEs have received much attention because they are used as a useful tool in the optimization field, such as the linear and quadratic programming, the optimal stopping in Markov chain, the structural mechanics, the network prices, the network equilibrium problems, the journal bearings, and the contact problems; see $[3,8,16,17,24,27,35$, $36,38]$ and the references therein.

The AVE is also equivalently reformulated to linear complementarity problem (LCP) and mixedinteger programming; see $[7,22,26]$ and the references therein. For example, consider the $\operatorname{LCP}(M, q)$ that contains of finding a vector $z \in R^{n}$, such that

$$
z \geqslant 0, \Omega=(M z+q) \geqslant 0, \quad z^{\top} \Omega=0,
$$

\footnotetext{
*Corresponding author

Email address: asad. adina@gmail.com (Asad Ali)

doi: $10.22436 /$ jmcs.026.04.01
} 
where $M \in R^{n \times n}$ and $q \in R^{n}$. The above LCP (1.2) can be reduced to the following AVE

$$
A x-B|x|=q,
$$

with

$$
x=\frac{1}{2}(B z+q)
$$

where $A=(M+I)$ and $B=(M-I)$. Abdallah et al. [1] transformed AVE (1.1) into a horizontal LCP, and solved it by a smoothing method. Prokopyev [31] has discussed the unique solvability of AVE, and its relation with LCP. Mangasarian [20] has shown that AVE is equivalent to a concave minimization problem and considered solving the latter problem instead of AVE. Hu and Huang [15] reformulated the AVE system to the standard LCP and provided some outcomes for the solution of AVE (1.1). Mezzadri [25] presented the equivalence between AVEs and horizontal LCPs.

In recent years, more and more studies have been conducted on the existence and uniqueness of the AVE solution and effective numerical solution algorithms of the AVE. For example, Hashemi and Ketabchi [14] proposed the numerical comparisons of smoothing functions for optimal correction of an infeasible system of AVEs. Saheya et al. [34] studied smoothing type algorithms for solving AVE (1.1) and proved that their algorithms have local and global quadratic convergence. Mangasarian [21] proposed an approximated generalized Newton method for solving AVE (1.1) and showed that this algorithm converges linearly from any initial point to the unique solution under the condition that $\left\|A^{-1}\right\|<\frac{1}{4}$. Caccetta et al. [5] studied a smoothing Newton method for solving AVE (1.1) and proved that the method is globally convergent and the convergence rate is quadratical under the weak condition that $\left\|A^{-1}\right\|<1$. Haghani [13] suggested the generalized Traub's method, which is better than the Mangasarian's method. Dong et al. [9] presented the SOR-like method for solving AVE (1.1). Nguyen et al. [30] presented unified smoothing functions associated with second-order cone for solving AVE (1.1). Gu et al. [12] suggested the nonlinear CSCS-like iteration method and the Picard-CSCS iteration method for solving AVE (1.1), which involves the toeplitz matrix. Moosaei et al. [28] showed that AVE (1.1) is equivalent to a bilinear programming problem. They solved the AVE by the principle of simulated annealing, and then found the minimum norm solution of AVE (1.1). Zhang et al. [37] introduced a new algorithm that relaxed the AVE into a convex optimization problem. They discovered the sparsest solution of the AVE through the minimum $l_{\infty}$-norm. Feng and Liu $[10,11]$ suggested and analyzed an improved generalized Newton method and two-step iterative method for solving AVE (1.1).

Recently, Li and Dai [19] and Najafi and Edalatpanah [29] presented different approaches using the fixed point principle to solve the LCPs. This research aims to extend this approach to AVE using the fixed point principle and formulate efficient iterative methods for solving AVE (1.1). The main contributions of this paper are given as follows. We split matrix $A$ into different parts and connect with Eq. (1.1) using different tools, which can speed up the convergence of the suggested iteration methods. Furthermore, we discuss the convergence of the novel methods under new conditions.

The rest of this paper is organized as follows. In Sec. 2, various notations and definitions are presented. In Sec. 3, we discuss the proposed methods and their convergence for solving AVE (1.1). Numerical results and concluding remarks are given in Secs. 4 and 5, respectively.

\section{Preparatory knowledge}

Here, we present some notations, the definition of $M$-matrix and a useful lemma for later analysis. Let $A=\left(a_{i j}\right) \in R^{n \times n}$, we write $A \geqslant 0$ if $a_{i j} \geqslant 0$ holds for all $1 \leqslant i, j \leqslant n$. We express the absolute value, spectral radius and norm of $A \in R^{n \times n}$ as $|A|=\left(\left|a_{i j}\right|\right), \rho(A)$ and $\|A\|_{\infty}$, respectively.

Definition 2.1 ([18]). Let $A \in R^{n \times n}$. It is called

1. a Z-matrix if $a_{i j} \leqslant 0$ for $i \neq j$;

2. an $M$-matrix if it is a non-singular $Z$-matrix satisfying $A^{-1} \geqslant 0$.

Lemma 2.2 ([23]). Let $A \in R^{n \times n}$, if $\left\|A^{-1}\right\|_{2}<1$, then the AVE (1.1) has a unique solution for any $b \in R^{n}$. 


\section{Proposed methods}

In this section, we discuss the suggested iterative methods. This section contains two parts. The first part includes the Jacobi, Gauss-Seidel, SOR methods and their convergence, and the second part includes the modified Picard method and their convergence for solving AVEs.

\subsection{Jacobi, Gauss-Seidel and SOR methods for AVE}

In this section, we discuss the Jacobi, Gauss-Seidel and SOR methods for solving AVEs. To propose and analyze the algorithms, we split the $A$ matrix as

$$
A=\widehat{D}_{A}-\widehat{L}_{A}-\widehat{U}_{A}
$$

where, $\widehat{D}_{A}, \widehat{L}_{A}$, and $\widehat{U}_{A}$ are the diagonal, the strictly lower-triangular and the strictly upper-triangular parts of $A$, respectively. If $A \in R^{n \times n}$ with $\operatorname{det}(A) \neq 0$, then the Jacobi, Gauss-Seidel, and SOR methods with relaxation factor $0<\lambda<2$, for solving AVE (1.1) are given respectively as,

$$
\begin{aligned}
x^{m+1} & =\widehat{D}_{A}^{-1}\left(\widehat{\mathrm{L}}_{A}+\widehat{\mathrm{U}}_{A}\right) x^{m}+\widehat{D}_{A}^{-1}\left(\left|x^{\mathrm{m}}\right|+b\right), \\
x^{m+1} & =\left(\widehat{\mathrm{D}}_{A}-\widehat{\mathrm{L}}_{A}\right)^{-1} \widehat{\mathrm{U}}_{A} x^{\mathrm{m}}+\left(\widehat{\mathrm{D}}_{A}-\widehat{\mathrm{L}}_{A}\right)^{-1}\left(\left|x^{\mathrm{m}}\right|+b\right), \\
x^{\mathrm{m}+1} & =\left(\widehat{\mathrm{D}}_{A}-\lambda \widehat{\mathrm{L}}_{A}\right)^{-1}\left[(1-\lambda) \widehat{\mathrm{D}}_{A}+\lambda \widehat{\mathrm{U}}_{A}\right] x^{\mathrm{m}}+\lambda\left(\widehat{\mathrm{D}}_{A}-\lambda \widehat{\mathrm{L}}_{A}\right)^{-1}\left(\left|x^{\mathrm{m}}\right|+b\right) .
\end{aligned}
$$

and the associated iteration matrices are

$$
H_{j}=\widehat{D}_{A}^{-1}\left(\widehat{L}_{A}+\widehat{U}_{A}\right), \quad H_{G S}=\left(\widehat{D}_{A}-\widehat{L}_{A}\right)^{-1} \widehat{U}_{A}, \quad H_{S O R}=\left(\widehat{D}_{A}-\lambda \widehat{L}_{A}\right)^{-1}\left[(1-\lambda) \widehat{D}_{A}+\lambda \widehat{U}_{A}\right] .
$$

It is well known that an iterative scheme $x^{\mathrm{m}+1}=\mathrm{H} x^{\mathrm{m}}+\mathrm{c}$ for solving AVE (1.1) converges for any initial guess $x^{(0)}$ of the solution if and only if $\rho(H)<1$ (see [4]). Therefore, in order to establish the convergence of the iteration methods defined in (3.1), it is sufficient to examine the spectral radius of the associated iteration matrix $\mathrm{H}$ in (3.2).

Theorem 3.1. Let $\mathrm{A}=\widehat{\mathrm{D}}_{\mathrm{A}}-\widehat{\mathrm{L}}_{\mathrm{A}}-\widehat{\mathrm{U}}_{\mathrm{A}}$, and $\chi^{(0)} \in \mathrm{R}^{\mathrm{n}}$ be an arbitrary initial guess. Then for $\rho\left(\widehat{\mathrm{D}}_{\mathrm{A}}^{-1}\left(\left|\widehat{\mathrm{L}}_{\mathrm{A}}+\widehat{\mathrm{U}}_{\mathrm{A}}\right|+\right.\right.$ I) $<1$, the iteration sequence $\left\{x^{\mathrm{m}}\right\}$ given by

$$
x^{m+1}=\widehat{D}_{A}^{-1}\left(\widehat{L}_{A}+\widehat{U}_{A}\right) x^{m}+\widehat{D}_{A}^{-1}\left(\left|x^{m}\right|+b\right),
$$

converges to the unique solution $\chi^{\star}$ of AVE (1.1).

Proof. Let $x^{\star}$ be a solution of AVE (1.1). Then

$$
x^{\star}=\widehat{D}_{A}^{-1}\left(\widehat{L}_{A}+\widehat{U}_{A}\right) x^{\star}+\widehat{D}_{A}^{-1}\left(\left|x^{\star}\right|+b\right) .
$$

After subtracting (3.4) from (3.3), we get

$$
x^{m+1}-x^{\star}=\widehat{D}_{A}^{-1}\left(\widehat{L}_{A}+\widehat{U}_{A}\right)\left(x^{m}-x^{\star}\right)+\widehat{D}_{A}^{-1}\left(\left|x^{m}\right|-\left|x^{\star}\right|\right) .
$$

From (3.5), we obtain

$$
\begin{aligned}
& \left|x^{m+1}-x^{\star}\right|=\left|\widehat{D}_{A}^{-1}\left(\widehat{L}_{A}+\widehat{U}_{A}\right)\left(x^{m}-x^{\star}\right)+\widehat{D}_{A}^{-1}\left(\left|x^{m}\right|-\left|x^{\star}\right|\right)\right| \leqslant \widehat{D}_{A}^{-1}\left|\widehat{L}_{A}+\widehat{U}_{A}\right|\left|x^{m}-x^{\star}\right|+\widehat{D}_{A}^{-1}|| x^{m}|-| x^{\star}||, \\
& \left|x^{m+1}-x^{\star}\right| \leqslant \widehat{D}_{A}^{-1}\left|\widehat{L}_{A}+\widehat{U}_{A}\right|\left|x^{m}-x^{\star}\right|+\widehat{D}_{A}^{-1}\left|x^{m}-x^{\star}\right|, \\
& \left|x^{m+1}-x^{\star}\right| \leqslant \widehat{D}_{A}^{-1}\left(\left|\widehat{L}_{A}+\widehat{U}_{A}\right|+I\right)\left|x^{m}-x^{\star}\right| .
\end{aligned}
$$

This shows that $\lim _{m \rightarrow \infty} x^{m}=x^{\star}$, when $\rho\left(\widehat{D}_{A}^{-1}\left(\left|\widehat{L}_{A}+\widehat{U}_{A}\right|+I\right)<1\right.$. 
For uniqueness of the solution, Let $\bar{x}$ is another solution of AVE. From the equations

$$
A x^{\star}-\left|x^{\star}\right|=b, \quad A \bar{x}-|\bar{x}|=b,
$$

written as

$$
x^{\star}=\widehat{D}_{A}^{-1}\left(\widehat{\mathrm{L}}_{\mathrm{A}}+\widehat{\mathrm{U}}_{\mathrm{A}}\right) \chi^{\star}+\widehat{\mathrm{D}}_{\mathrm{A}}^{-1}\left(\left|x^{\star}\right|+\mathrm{b}\right), \quad \overline{\mathrm{x}}=\widehat{\mathrm{D}}_{\mathrm{A}}^{-1}\left(\widehat{\mathrm{L}}_{\mathrm{A}}+\widehat{\mathrm{U}}_{\mathrm{A}}\right) \overline{\mathrm{x}}+\widehat{\mathrm{D}}_{\mathrm{A}}^{-1}(|\bar{x}|+\mathrm{b}),
$$

we obtain

$$
\left|x^{\star}-\bar{x}\right| \leqslant\left(\widehat{D}_{A}^{-1}\left(\left|\widehat{L}_{A}+\widehat{U}_{A}\right|+I\right)\left|x^{\star}-\bar{x}\right| .\right.
$$

Since $\rho\left(\widehat{D}_{A}^{-1}\left(\left|\widehat{L}_{A}+\widehat{U}_{A}\right|+I\right)<1\right.$, we have $x^{\star}=\bar{x}$. The proof is completed.

Theorem 3.2. Suppose that $A V E(1.1)$ is solvable and $A=\widehat{D}_{A}-\widehat{L}_{A}-\widehat{U}_{A}$, be the splitting of $A$. If

$$
\left|x^{m+1}-x^{\star}\right| \leqslant\left|\left(\widehat{D}_{A}-\widehat{L}_{A}\right)^{-1}\right|\left(\left|\widehat{U}_{A}\right|+I\right)\left|x^{m}-x^{\star}\right|,
$$

then for any initial vector $x^{(0)} \in \mathrm{R}^{n}$, the Gauss-Seidel method converges to the unique solution $x^{\star}$ of AVE (1.1).

Proof. Let $x^{\star}$ be a solution of AVE (1.1). Then

$$
x^{\star}=\left(\widehat{D}_{A}-\widehat{L}_{A}\right)^{-1} \widehat{U}_{A} x^{\star}+\left(\widehat{D}_{A}-\widehat{L}_{A}\right)^{-1}\left(\left|x^{\star}\right|+b\right) .
$$

After subtracting (3.6) from (3.1), we get

$$
x^{m+1}-x^{\star}=\left(\widehat{D}_{A}-\widehat{L}_{A}\right)^{-1} \widehat{U}_{A}\left(x^{m}-x^{\star}\right)+\left(\widehat{D}_{A}-\widehat{L}_{A}\right)^{-1}\left(\left|x^{m}\right|-\left|x^{\star}\right|\right) .
$$

From (3.7), we obtain

$$
\begin{aligned}
\left|x^{m+1}-x^{\star}\right| & =\left|\left(\widehat{D}_{A}-\widehat{\mathrm{L}}_{A}\right)^{-1} \widehat{\mathrm{U}}_{A}\left(x^{\mathrm{m}}-x^{\star}\right)+\left(\widehat{\mathrm{D}}_{A}-\widehat{\mathrm{L}}_{A}\right)^{-1}\left(\left|x^{\mathrm{m}}\right|-\left|x^{\star}\right|\right)\right| \\
& \leqslant\left|\left(\widehat{\mathrm{D}}_{A}-\widehat{\mathrm{L}}_{A}\right)^{-1}\right|\left|\widehat{\mathrm{U}}_{A}\right|\left|x^{\mathrm{m}}-x^{\star}\right|+\left|\left(\widehat{\mathrm{D}}_{A}-\widehat{\mathrm{L}}_{A}\right)^{-1}\right||| x^{\mathrm{m}}|-| x^{\star}||, \\
\left|x^{\mathrm{m}+1}-x^{\star}\right| & \leqslant\left|\left(\widehat{\mathrm{D}}_{A}-\widehat{\mathrm{L}}_{A}\right)^{-1}\right|\left|\widehat{\mathrm{U}}_{A}\right|\left|x^{\mathrm{m}}-x^{\star}\right|+\left|\left(\widehat{\mathrm{D}}_{A}-\widehat{\mathrm{L}}_{A}\right)^{-1}\right|\left|x^{\mathrm{m}}-x^{\star}\right|, \\
\left|x^{\mathrm{m}+1}-x^{\star}\right| & \leqslant\left|\left(\widehat{D}_{A}-\widehat{\mathrm{L}}_{A}\right)^{-1}\right|\left(\left|\widehat{\mathrm{U}}_{A}\right|+\mathrm{I}\right)\left|x^{\mathrm{m}}-x^{\star}\right| .
\end{aligned}
$$

Note that the matrix $\left|\left(\widehat{D}_{A}-\widehat{L}_{A}\right)^{-1}\right|\left(\left|\widehat{U}_{A}\right|+I\right)$ is non-negative. We know that in $\left[2\right.$, Theorem 4.1], if $\rho\left(\mid\left(\widehat{D}_{A}-\right.\right.$ $\left.\left.\widehat{\mathrm{L}}_{A}\right)^{-1} \mid\left(\left|\widehat{\mathrm{U}}_{A}\right|+\mathrm{I}\right)\right)<1$, then the sequence $\left\{x^{\mathrm{m}}\right\}$ of the Gauss-Seidel method converges to the solution $x^{\star}$ of AVE.

The proof of the uniqueness is easy and is omitted here. The uniqueness of the solution, follows the same steps as Theorem 3.1.

Theorem 3.3. Suppose that $A V E(1.1)$ is solvable and $A=\widehat{D}_{A}-\widehat{L}_{A}-\widehat{U}_{A}$, be the splitting of $A$. If

$$
\left|x^{m+1}-x^{\star}\right| \leqslant\left|\left(\widehat{D}_{A}-\lambda \widehat{L}_{A}\right)^{-1}\right|\left(\left|(1-\lambda) \widehat{D}_{A}+\lambda \widehat{U}_{A}\right|+\lambda\right)\left|x^{m}-x^{\star}\right|,
$$

then for any initial vector $x^{(0)} \in \mathrm{R}^{n}$, the SOR method converges to the unique solution $x^{\star}$ of AVE (1.1).

Proof. Let $x^{\star}$ be a solution of AVE (1.1). Then

$$
x^{\star}=\left(\widehat{\mathrm{D}}_{\mathrm{A}}-\lambda \widehat{\mathrm{L}}_{\mathrm{A}}\right)^{-1}\left[(1-\lambda) \widehat{\mathrm{D}}_{\mathrm{A}}+\lambda \widehat{\mathrm{U}}_{\mathrm{A}}\right] x^{\star}+\lambda\left(\widehat{\mathrm{D}}_{\mathrm{A}}-\lambda \widehat{\mathrm{L}}_{\mathrm{A}}\right)^{-1}\left(\left|x^{\star}\right|+b\right) .
$$

After subtracting (3.8) from (3.1), we get

$$
x^{m+1}-x^{\star}=\left(\widehat{D}_{A}-\lambda \widehat{\mathrm{L}}_{A}\right)^{-1}\left[(1-\lambda) \widehat{\mathrm{D}}_{A}+\lambda \widehat{\mathrm{U}}_{A}\right]\left(x^{\mathrm{m}}-x^{\star}\right)+\lambda\left(\widehat{\mathrm{D}}_{A}-\lambda \widehat{\mathrm{L}}_{A}\right)^{-1}\left(\left|x^{\mathrm{m}+1}\right|-\left|x^{\star}\right|\right) .
$$


From (3.9), we obtain

$$
\begin{aligned}
& \left|x^{m+1}-x^{\star}\right|=\left|\left(\widehat{\mathrm{D}}_{A}-\lambda \widehat{\mathrm{L}}_{A}\right)^{-1}\left[(1-\lambda) \widehat{\mathrm{D}}_{A}+\lambda \widehat{\mathrm{U}}_{A}\right]\left(x^{m}-x^{\star}\right)+\lambda\left(\widehat{\mathrm{D}}_{A}-\lambda \widehat{\mathrm{L}}_{A}\right)^{-1}\left(\left|x^{m}\right|-\left|x^{\star}\right|\right)\right| \\
& \leqslant\left|\left(\widehat{\mathrm{D}}_{A}-\lambda \widehat{\mathrm{L}}_{A}\right)^{-1}\right|\left|(1-\lambda) \widehat{\mathrm{D}}_{A}+\lambda \widehat{\mathrm{U}}_{A}\right|\left|x^{\mathrm{m}}-x^{\star}\right|+\lambda\left|\left(\widehat{\mathrm{D}}_{A}-\lambda \widehat{\mathrm{L}}_{A}\right)^{-1}\right|\left|x^{\mathrm{m}}\right|-\left|x^{\star}\right| \mid, \\
& \left|x^{m+1}-x^{\star}\right| \leqslant\left|\left(\widehat{D}_{A}-\lambda \widehat{L}_{A}\right)^{-1}\right|\left|(1-\lambda) \widehat{D}_{A}+\lambda \widehat{U}_{A}\right|\left|x^{m}-x^{\star}\right|+\lambda\left|\left(\widehat{D}_{A}-\lambda \widehat{L}_{A}\right)^{-1}\right|\left|x^{m}-x^{\star}\right|, \\
& \left|x^{m+1}-x^{\star}\right| \leqslant\left|\left(\widehat{D}_{A}-\lambda \widehat{L}_{A}\right)^{-1}\right|\left(\left|(1-\lambda) \widehat{D}_{A}+\lambda \widehat{U}_{A}\right|+\lambda\right)\left|x^{m}-x^{\star}\right| .
\end{aligned}
$$

Evidently, if $\rho\left(\left|\left(\widehat{D}_{A}-\lambda \widehat{L}_{A}\right)^{-1}\right|\left(\left|(1-\lambda) \widehat{D}_{A}+\lambda \widehat{U}_{A}\right|+\lambda\right)\right)<1$, the iteration sequence $\left\{x^{m}\right\}$ generated by the SOR method converges to the solution $\chi^{\star}$ of AVE (1.1).

The uniqueness of the solution, follows the same steps as Theorem 3.1.

\subsection{Modified Picard method}

Rohn et al. [33] proposed the Picard iteration method for solving AVEs. This method can be summarized as follows:

$$
x^{m+1}=A^{-1}\left(\left|x^{m}\right|+b\right), \quad m=0,1,2, \ldots
$$

In this section, we promote this idea and suggest the modified Picard iterative method for solving AVEs.

Modified Picard method. Let $A$ be a non-singular matrix of order $n \times n$ and $b$ be a vector of order $n$. Given an initial vector $x^{0} \in R^{n}$ and for $m=0,1,2, \ldots$, until the iteration sequence $\left\{x^{m}\right\}_{m=0}^{\infty}$ is convergent, compute

$$
\left\{\begin{array}{l}
x^{m+\frac{1}{2}}=A^{-1}\left|x^{m}\right|+A^{-1} b \\
x^{m+1}=A^{-1}\left|x^{m+\frac{1}{2}}\right|+A^{-1} b .
\end{array}\right.
$$

Now, the following theorem indicates the convergence of the proposed method.

Theorem 3.4. Suppose that (1.1) is solvable and $A \in \mathrm{R}^{\mathrm{n} \times \mathrm{n}}$ satisfies Lemma 2.2. If

$$
\left|x^{m+1}-x^{\star}\right| \leqslant\left|A^{-1}\right|^{2}\left|x^{m}-x^{\star}\right|,
$$

then, for any initial vector $x^{(0)} \in \mathrm{R}^{\mathrm{n}}$ the sequence $\left\{x^{\mathrm{m}}\right\}$ generated by the modified Picard method converges to the unique solution $x^{\star} \in \mathrm{R}^{\mathrm{n}}$ of the AVE (1.1).

Proof. Consider $x^{\star}$ is the solution of AVE (1.1), then we have

$$
\begin{aligned}
& \left\{\begin{array}{l}
x^{m+\frac{1}{2}}-x^{\star}=\left(A^{-1}\left|x^{m}\right|+A^{-1} b\right)-\left(A^{-1}\left|x^{\star}\right|+A^{-1} b\right), \\
x^{m+1}-x^{\star}=\left(A^{-1}\left|x^{m+\frac{1}{2}}\right|+A^{-1} b\right)-\left(A^{-1}\left|x^{\star}\right|+A^{-1} b\right),
\end{array}\right. \\
& \left\{\begin{array}{l}
x^{m+\frac{1}{2}}-x^{\star}=A^{-1}\left(\left|x^{m}\right|-\left|x^{\star}\right|\right) \\
x^{m+1}-x^{\star}=A^{-1}\left(\left|x^{m+\frac{1}{2}}\right|-\left|x^{\star}\right|\right) .
\end{array}\right.
\end{aligned}
$$

By taking the first part of (3.10) and using absolute values on both sides, we get

$$
\left|x^{m+\frac{1}{2}}-x^{\star}\right| \leqslant\left|A^{-1}\right||| x^{m}|-| x^{\star}||, \quad\left|x^{m+\frac{1}{2}}-x^{\star}\right| \leqslant\left|A^{-1} \| x^{m}-x^{\star}\right| .
$$

Similarly, we get from the second part in (3.10) that

$$
\left|x^{m+1}-x^{\star}\right| \leqslant\left|A^{-1}\right|\left|x^{m+\frac{1}{2}}-x^{\star}\right| .
$$

From (3.11) and (3.12), we obtain

$$
\left|x^{m+1}-x^{\star}\right| \leqslant\left|A^{-1}\right| \times\left|A^{-1}\right|\left|x^{m}-x^{\star}\right| .
$$

So,

$$
\left|x^{m+1}-x^{\star}\right| \leqslant\left|A^{-1}\right|^{2}\left|x^{m}-x^{\star}\right| .
$$

Note that the matrix $\left|A^{-1}\right|^{2}$ is non-negative. We know if $\rho\left(\left|A^{-1}\right|^{2}\right)<1$, then the sequence $\left\{x^{m}\right\}$ of the proposed method converges to the unique solution $x^{\star}$ of AVE. This completes the proof. 


\section{Numerical experiments}

Now, we consider some numerical examples to show the performance of the new methods. All computations are performed on Intel (C) Core (TM) i7-10875H, where the CPU is $5.1 \mathrm{GHz}$, the memory is 32 GB, and MATLAB (2017a) programming language. All tests are started from the initial zero vector and the termination condition is given by

$$
\text { RES }:=\frac{\left\|A x^{m}-\left|x^{m}\right|-b\right\|_{2}}{\|b\|_{2}} \leqslant 10^{-6},
$$

where $\|x\|$ denotes the 2-norm. To computationally study the performance of the proposed methods, we examine the following two examples.

Example 4.1 ([6]). Let the AVE (1.1) with $A=M+\Psi I \in R^{n \times n}$ and $b=A x-|x| \in R^{n}$, such that

$$
\mathrm{M}=\left(\begin{array}{ccccc}
\mathrm{S} & -\mathrm{I} & & \\
-\mathrm{I} & \mathrm{S} & -\mathrm{I} & & \\
& -\mathrm{I} & \mathrm{S} & \ddots & \\
& & \ddots & \ddots & -\mathrm{I} \\
& & & -\mathrm{I} & \mathrm{S}
\end{array}\right) \in \mathrm{R}^{\mathrm{n} \times \mathrm{n}}, \quad \mathrm{x}=(-1,1,-1,1, \ldots,-1,1)^{\top} \in \mathrm{R}^{\mathrm{n}},
$$

where $S=\operatorname{tridiag}(-1,4,-1) \in \mathrm{R}^{v \times v}, \mathrm{I} \in \mathrm{R}^{v \times v}$ is the identity matrix, $\Psi=4$ and $\mathrm{n}=v^{2}$. The numerical results are listed in Table 1.

Table 1: Numerical results of Example 4.1 with $\lambda=1.2$.

\begin{tabular}{llllll}
\hline Methods & $\mathrm{n}$ & 64 & 256 & 1024 & 4096 \\
\hline JM & Iter & 32 & 40 & 43 & 44 \\
& Time & 0.0136 & 0.0386 & 0.0683 & 0.2466 \\
& RES & $9.10 \mathrm{e}-07$ & $8.54 \mathrm{e}-07$ & $9.04 \mathrm{e}-07$ & $9.32 \mathrm{e}-07$ \\
GSM & Iter & 20 & 25 & 27 & 27 \\
& Time & 0.00112 & 0.0174 & 0.0358 & 0.1162 \\
& RES & $9.11 \mathrm{e}-07$ & $7.19 \mathrm{e}-07$ & $7.32 \mathrm{e}-07$ & $9.73 \mathrm{e}-07$ \\
SORM & Iter & 16 & 17 & 18 & 19 \\
& Time & 0.0082 & 0.099 & 0.0158 & 0.1102 \\
& RES & $5.15 \mathrm{e}-07$ & $7.20 \mathrm{e}-07$ & $9.73 \mathrm{e}-07$ & $6.94 \mathrm{e}-07$ \\
MPM & Iter & 5 & 6 & 6 & 6 \\
& Time & 0.0035 & 0.0068 & 0.0089 & 0.0348 \\
& RES & $6.46 \mathrm{e}-07$ & $1.47 \mathrm{e}-07$ & $2.41 \mathrm{e}-07$ & $2.88 \mathrm{e}-07$ \\
\hline
\end{tabular}

In Table 1, we list the number of iterations (Iter), the CPU times in seconds (Time), and the 2-norm of residual vectors (RES) of all methods. Moreover, we denote the Jacobi Method by JM, the Gauss-Seidel method by GSM, the SOR method by SORM, and modified Picard method by MPM. From the numerical results in Table 1, we notice that the suggested methods can rapidly calculate the AVE solution under some conditions.

Example 4.2 ([6]). Let

$$
A=\left(\begin{array}{ccccc}
8 & -1 & & & \\
-1 & 8 & -1 & & \\
& -1 & 8 & \ddots & \\
& & \ddots & \ddots & -1 \\
& & & -1 & 8
\end{array}\right) \in R^{n \times n}, \quad x=(-1,1,-1,1, \ldots,-1,1)^{\top} \in R^{n}
$$

and $b=A x-|x| \in R^{n}$. The numerical results are discussed in Table 2. 
Table 2: Numerical results of Example 4.2 with $\lambda=0.98$.

\begin{tabular}{llllll}
\hline Methods & $\mathrm{n}$ & 1000 & 2000 & 3000 & 4000 \\
\hline JM & Iter & 11 & 11 & 11 & 11 \\
& Time & 0.0024 & 0.0078 & 0.0087 & 0.0120 \\
& RES & $4.13 \mathrm{e}-09$ & $5.84 \mathrm{e}-09$ & $7.15 \mathrm{e}-09$ & $8.26 \mathrm{e}-09$ \\
GSM & Iter & 8 & 8 & 8 & 8 \\
& Time & 0.0015 & 0.0033 & 0.0075 & 0.0089 \\
& RES & $6.27 \mathrm{e}-09$ & $9.51 \mathrm{e}-09$ & $2.59 \mathrm{e}-09$ & $3.00 \mathrm{e}-09$ \\
SORM & Iter & 9 & 9 & 9 & 9 \\
& Time & 0.0019 & 0.0054 & 0.077 & 0.0102 \\
& RES & $6.27 \mathrm{e}-09$ & $9.51 \mathrm{e}-09$ & $2.59 \mathrm{e}-09$ & $3.00 \mathrm{e}-09$ \\
MPM & Iter & 4 & 4 & 4 & 4 \\
& Time & 0.0011 & 0.0017 & 0.0037 & 0.0067 \\
& RES & $1.06 \mathrm{e}-09$ & $1.50 \mathrm{e}-09$ & $1.84 \mathrm{e}-09$ & $2.12 \mathrm{e}-09$ \\
\hline
\end{tabular}

From Table 2, all tested methods can quickly calculate the solution of AVE (1.1). However, the computing time and the number of iterations of the MPM method is less than the other methods. Finally, we conclude that our proposed methods are feasible and effective for AVEs.

\section{Conclusion}

In this work, we dealt with some iterative methods for solving AVE and studied suitable cases to converge the recommended iterative methods. These methods are easy to implement in practice. In addition, some numerical results have shown the effectiveness of these methods. Numerical results show that the proposed schemes are suitable for large and sparse AVE.

This paper successfully examined the new iterative methods for determining AVE (1.1) when the coefficient matrix is an M-matrix. The cases for more general coefficient matrices and comparison with other new iteration methods are the next issue to be considered.

\section{Acknowledgments}

The authors would like to thank the three referees and the editor for their careful reading and valuable comments on improving the original manuscript.

\section{References}

[1] L. Abdallah, M. Haddou, T. Migot, Solving absolute value equation using complementarity and smoothing functions, J. Comput. Appl. Math., 327 (2018), 196-207. 1

[2] B. H. Ahn, Solution of nonsymmetric linear complementarity problems by iterative methods, J. Optim. Theory Appl., 33 (1981), 175-185. 3.1

[3] R. Ali, M. R. Khan, A. Abidi, S. Rasheed, A. M. Galal, Application of PEST and PEHF in magneto-Williamson nanofluid depending on the suction/injection, Case Stud. Thermal Eng., 27 (2021), 11 pages. 1

[4] A. Berman, R. J. Plemmons, Nonnegative Matrices in the Mathematical Science, SIAM, Philadelphia, (1994). 3.1

[5] L. Caccetta, B. Qu, G.-L. Zhou, A globally and quadratically convergent method for absolute value equations, Comput. Optim. Appl., 48 (2011), 45-58. 1

[6] C. Chen, D. Yu, D. Han, Optimal parameter for the SOR-like iteration method for solving the system of absolute value equations, arXiv, 2020 (2020), 23 pages 4.1, 4.2

[7] R. W. Cottle, J.-S. Pang, R. E. Stone, The Linear Complementarity Problem, SIAM, Philadelphia, (2009). 1

[8] M. Dehghan, A. Shirilord, Matrix multisplitting Picard-iterative method for solving generalized absolute value matrix equation, Appl. Numer. Math., 158 (2020), 425-438. 1

[9] J.-L. Dong, M.-Q. Jiang, A modified modulus method for symmetric positive-definite linear complementarity problems, Numer. Linear Algebra Appl., 16 (2009), 129-143. 1 
[10] J.-M. Feng, Y.-S. Liu, An improved generalized Newton method for absolute value equations, SpringerPlus, 5 (2016), 10 pages. 1

[11] J. M. Feng, Y. S. Liu, A new two-step iterative method for solving absolute value equations, J. Inequal. Appl., 2019 (2019), 8 pages. 1

[12] X. M. Gu, T. Z. Huang, H. B. Li, S. F. Wang, L. Li, Two-CSCS based iteration methods for solving absolute value equations, J. Appl. Math. Comput., 7 (2017), 1336-1356. 1

[13] F. K. Haghani, On generalized Traub's method for absolute value equations, J. Optim. Theory Appl., 166 (2015), 619-625. 1

[14] F. Hashemi, S. Ketabchi, Numerical comparisons of smoothing functions for optimal correction of an infeasible system of absolute value equations, Numer. Algebra Control Optim., 10 (2020), 13-21. 1

[15] S.-L. Hu, Z.-H. Huang, A note on absolute value equations, Optim. Lett., 4 (2010), 417-424. 1

[16] Y.-F. Ke, C.-F. Ma, SOR-like iteration method for solving absolute value equations, Appl. Math. Comput., 311 (2017), 195-202. 1

[17] M. R. Khan, M. Li, S. Mao, R. Ali, S. Khan, Comparative study on heat transfer and friction drag in the flow of various hybrid nanofluids efiected by aligned magnetic fleld and nonlinear radiation, Sci. Rep., 11 (2021), 1-14. 1

[18] C.-X. Li, A preconditioned AOR iterative method for the absolute value equations, Int. J. Comput. Methods, 14 (2017), 12 pages. 2.1

[19] Y. T. Li, P. F. Dai, Generalized AOR methods for linear complementarity problem, Appl. Math. Comput., 188 (2007), 7-18. 1

[20] O. L. Mangasarian, Absolute value equation solution via concave minimization, Optim. Lett., 1 (2007), 3-8. 1

[21] O. L. Mangasarian, A generalized Newton method for absolute value equations, Optim. Lett., 3 (2009), 101-108. 1

[22] O. L. Mangasarian, Linear complementarity as absolute value equation solution, Optim. Lett., 8 (2014), 1529-1534. 1

[23] O. L. Mangasarian, R. R. Meyer, Absolute value equation, Linear Algebra Appl., 419 (2006), 359-367. 1, 2.2

[24] A. Mansoori, M. Erfanian, A dynamic model to solve the absolute value equations, J. Comput. Appl. Math., 333 (2018), 28-35. 1

[25] F. Mezzadri, On the solution of general absolute value equations, Appl. Math. Lett., 107 (2020), 7 pages. 1

[26] F. Mezzadri, E. Galligani, Modulus-based matrix splitting methods for horizontal linear complementarity problems, Numer. Algorithms, 83 (2020), 201-219. 1

[27] X.-H. Miao, J.-T. Yang, B. Saheya, J.-S. Chen, A smoothing Newton method for absolute value equation associated with second-order cone, Appl. Numer. Math., 120 (2017), 82-96. 1

[28] H. Moosaei, S. Ketabchi, H. Jafari, Minimum norm solution of the absolute value equations via simulated annealing algorithm, Afr. Math., 26 (2015), 1221-1228. 1

[29] H. S. Najafi, S. A. Edalatpanah, SOR-like methods for non-Hermitian positive definite linear complementarity problems, Adv. Model. Optim., 15 (2013), 697-704. 1

[30] C. T. Nguyen, B. Saheya, Y.-L. Chang, J.-S. Chen, Unified smoothing functions for absolute value equation associated with second-order cone, Appl. Numer. Math., 135 (2019), 206-227. 1

[31] O. Prokopyev, On equivalent reformulations for absolute value equations, Comput. Optim. Appl., 44 (2009), 363-372. 1

[32] J. Rohn, A theorem of the alternatives for the equation $A x+B|x|=b$, Linear Multilinear Algebra, 52 (2004), 421-426. 1

[33] J. Rohn, V. Hooshyarbakhsh, R. Farhadsefat, An iterative method for solving absolute value equations and sufficient conditions for unique solvability, Optim. Lett., 8 (2014), 35-44. 3.2

[34] B. Saheya, C.-H. Yu, J.-S. Chen, Numerical comparisons based on four smoothing functions for absolute value equation, J. Appl. Math. Comput., 56 (2018), 131-149. 1

[35] I. Uddin, I. Ullah, R. Ali, I. Khan, K.S. Nisar, Numerical analysis of nonlinear mixed convective MHD chemically reacting flow of Prandtl-Eyring nanofluids in the presence of activation energy and Joule heating, J. Therm. Anal. Calorim., 145 (2021), 495-505. 1

[36] I. Ullah, R. Ali, I. Khan, K.S. Nisar, Insight into kerosene conveying CNTs and Fe3O4 nanoparticles through a porous medium: signiflcance of Coriolis force and entropy generation, Phys. Scr., 96 (2021), 14 pages. 1

[37] M. Zhang, Z.-H. Huang, Y.-F. Li, The sparsest solution to the system of absolute value equations, J. Oper. Res. Soc. China, 3 (2015), 31-51. 1

[38] T. H. Zhao, M. R. Khan, Y. M. Chu, A. Issakhov, R. Ali, S. Khan, Entropy generation approach with heat and mass transfer in magnetohydrodynamic stagnation point flow of a tangent hyperbolic nanofluid, Appl. Math. Mech., 42 (2021), 1205-1218. 1 